\title{
Electron Tomographic Characterization of Nano-Confined Hydrides for Hydrogen Storage
}

\author{
S.D. House*, E.H. Majzoub ${ }^{\dagger}$, R.K. Bhakta ${ }^{\ddagger}$, M.D. Allendorf ${ }^{\ddagger}$, and I.M. Robertson* \\ *Department of Materials Science and Engineering, University of Illinois at Urbana-Champaign, \\ Urbana, IL, 61801 \\ ${ }^{\dagger}$ Center for Nanoscience and Department of Physics and Astronomy, University of Missouri-St. \\ Louis, St. Louis, MO, 63121 \\ ${ }^{*}$ Sandia National Laboratory, Livermore, CA, 94551-0969
}

Complex metal hydrides are promising materials for automotive hydrogen storage. Their high sorption temperatures for uptake and release of hydrogen must be addressed before they can be employed in such applications. Reducing the particle size to the nanoscale, preferably to below 10 $\mathrm{nm}[1]$, has the potential to address these challenges through decreased diffusion distances, increased surface area, and increased interfacial contract between phases. However, the particle size must be retained through many cycles. Confinement of storage materials within nanoporous scaffolds offers a possible solution as it constrains particle dimensions to that of the host pore; restricts long-range diffusion of elements following dehydrogenation, which limits phase segregation and maintains interfacial contact between the reacting phases; and inhibits sintering of hydride nanoparticles, which would otherwise result in the loss of nanoscale particles with cycling.

Optimization of system choice and performance requires knowledge of the relationship between the processing conditions used to synthesize these systems and their resulting microstructure (3D structure, pore size, wall thickness, and connectivity), the penetration of the storage medium, and their cyclic stability (structural, morphological, and composition), and so to their performance. As the three-dimensionality of these systems is crucial to their understanding, conventional 2D imaging techniques cannot provide unambiguous answers to these questions. Electron tomography provides 3D structural and compositional information and is well suited to investigate these systems.

To test the viability of applying electron tomography to nano-confined systems, Ag-infiltrated MOF508 metal-organic frameworks[2] were imaged using high-angle annular dark-field (HAADF) transmission electron microscopy. From this data, 3D tomograms were reconstructed, Figure 1, in which the infiltrated nano-particles of Ag were clearly distinguishable from the MOF scaffold. Unlike conventional 2D images of this system, the tomograms conclusively show that the $\mathrm{Ag}$ particle were uniformly dispersed throughout the interior of the framework and not agglomerated on the surface. Using this approach, the incorporation of sodium alanate in a $\mathrm{Cu}_{3}(\mathrm{BTC})_{2}$ framework has been demonstrated.

Nano-porous carbon scaffolds are being explored as potential storage structure but there has been no attempt to correlate the synthesis parameters with the resulting structures. Although the scaffold is nominally composed of thin columns packed in a hexagonal arrangement, the actual microstructure is much more complicated. For example, the particles that constitute the scaffold are subdivided into domains, in each of which the columns are in different directions, which might mean that not all pores are accessible; see, for example, Figure 2a. Even in what appear to be a single domain, the columns do not always have the same angular orientation, Figure $2 \mathrm{~b}$. Additionally, the same domain 
can have not only different sizes but different curvatures of columns as well, Figure $2 c-d$. Furthermore, not all columns are continuous some are pinched-off along the length. The impact of having a range of structures on infiltration of a storage medium will be discussed.

This work was partially supported through the Metal Hydrides Center of Excellence, Office of Energy Efficiency and Renewable Energy, U.S. Department of Energy under grant No. DE-FC3605GO15064. The authors acknowledge the use of the facilities in the Center of Microanalysis of Materials at the University of Illinois. IMR acknowledges support from the National Science Foundation, while working at the Foundation.

\section{References}

[1] M. Fichtner, Nanotechnology, 2009. 20 (20)

[2] R.J.T. Houk, et al., Nano Letters, 2009. 9 (10)

[3] Funding was provided through DOE grant DE-FC36-05GO15064

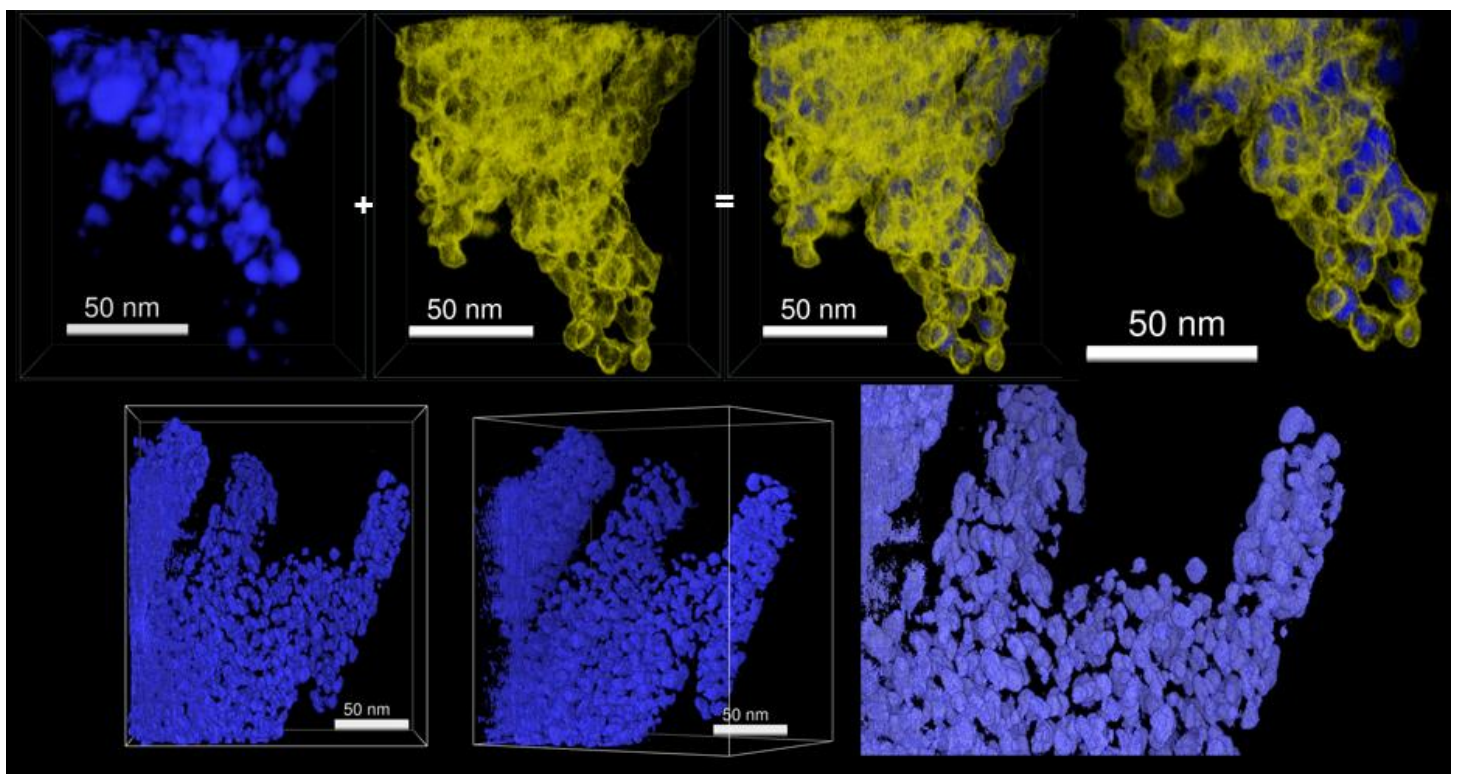

FIG 1. Tomographic reconstructions of Ag nanoparticles (blue) infiltrated into a MOF-508 framework (yellow). The framework has been removed on the lower reconstruction for clarity.

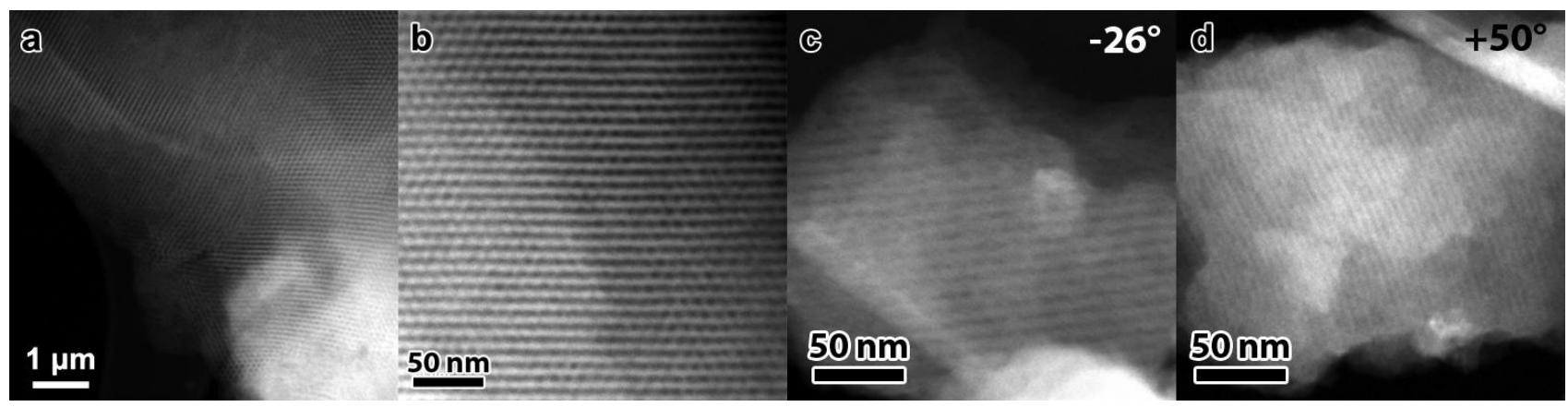

FIG 2. HAADF-STEM images of nano-porous carbon showing (a) small domains of different column orientations within a larger powder grain, and (b) column layers with hexagonal angular offsets to those below them. (c-d) HAADF-STEM images of a grain taken at $-26^{\circ}$ and $+50^{\circ}$ of tilt, respectively, showing differences in column orientation, size, and curvature. 